\title{
CPA Epidermoid Cyst with Rare Anatomic Variant: Anterior Inferior Cerebellar Artery Embedded in the Subarcuate Fossa: Operative Video and Technical Nuances
}

\author{
Carlos Candanedo ${ }^{1}$ Sergey Spektor ${ }^{1}$ \\ ${ }^{1}$ Department of Neurosurgery, Hadassah-Hebrew University Medical \\ Center, Jerusalem, Israel \\ J Neurol Surg B 2019;80(suppl S3):S323-S324.
}

Address for correspondence Carlos Candanedo, MD, Department of Neurosurgery, Hadassah Hebrew University Medical Center, P.O. Box 12000, Jerusalem, Israel 91120

(e-mail: ccandanedomd@hotmail.com).
Intracranial epidermoid cysts are considered benign tumors with a good general prognosis; however, their radical removal, including tumor capsule, is associated with significant morbidity, especially when the capsule is attached to neurovascular structures. We show an operative video describing main steps and surgical nuances in the resection of a large right cerebellopontine angle (CPA) epidermoid cyst in a 42-year-old male patient who presented with intractable trigeminal neuralgia. Craniectomy was performed to exposure the transverse-sigmoid sinus junction. A mold for a polymethylmethacrylate (PMMA) bone flap was built before opening the dura to avoid potentially neurotoxic effects on the cerebellum. The video illustrates the management of the rare anatomical variant of the anterior inferior cerebellar artery (AICA). Its loop was embedded in the dura, covering the subarcuate fossa where it gives off the subarcuate artery. Near total removal of the epidermoid cyst was achieved, leaving only a tiny capsule remnant adhering to the abducens nerve. Postoperatively the patient's trigeminal neuralgia was fully relieved and medications were discontinued. The patient's hearing was preserved per audiometry at the preoperative level (Gardner-Robertson II). Postoperative magnetic resonance imaging (MRI) revealed no signs of residual tumor. In this case, it was not possible to obtain optimal surgical exposure of the CPA without handling a rare anatomical anomaly of the AICA in the dura of the subarcuate fossa, which demanded coagulation and transection of the subarcuate artery and transposition of AICA with the dural cuff. This manipulation enabled optimal surgical removal of the epidermoid and didn't cause any neurological deficit. The link to the video can be found at: https://youtu.be/ILZqBHlu-uA.
Conflict of Interest

None.

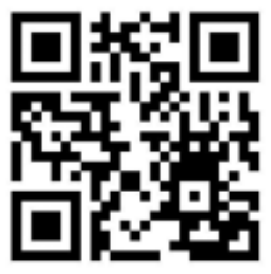

received

May 31, 2018

accepted

August 18, 2018

published online

October 18, 2018

www.thieme.com/skullbasevideos

www.thieme.com/jnlsbvideos

DOI https://doi.org/

10.1055/s-0038-1675165. ISSN 2193-6331.
๑) 2019 Georg Thieme Verlag KG
Stuttgart · New York

License terms

c) $(1) \$$ 


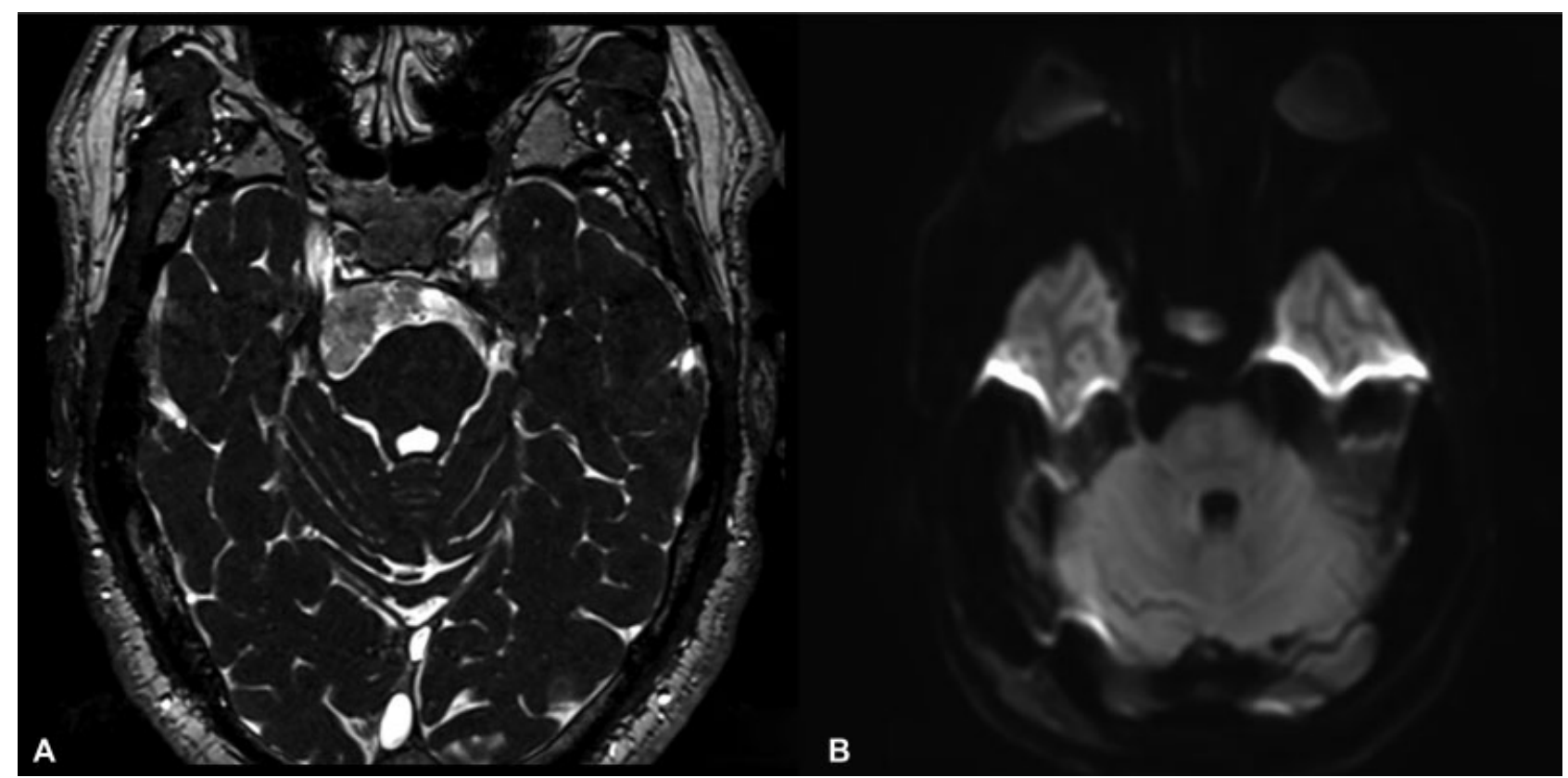

Fig. 1 Pre- and postoperative magnetic resonance imaging (MRI). (A) Preoperative axial 3D gradient echo steady-state sequence MRI showing a right cerebellopontine angle (CPA) epidermoid cyst pushing posterolaterally the right trigeminal nerve. (B) Postoperative axial diffusionweighted imaging (DWI) showing no signs of epidermoid cyst tumor residual.

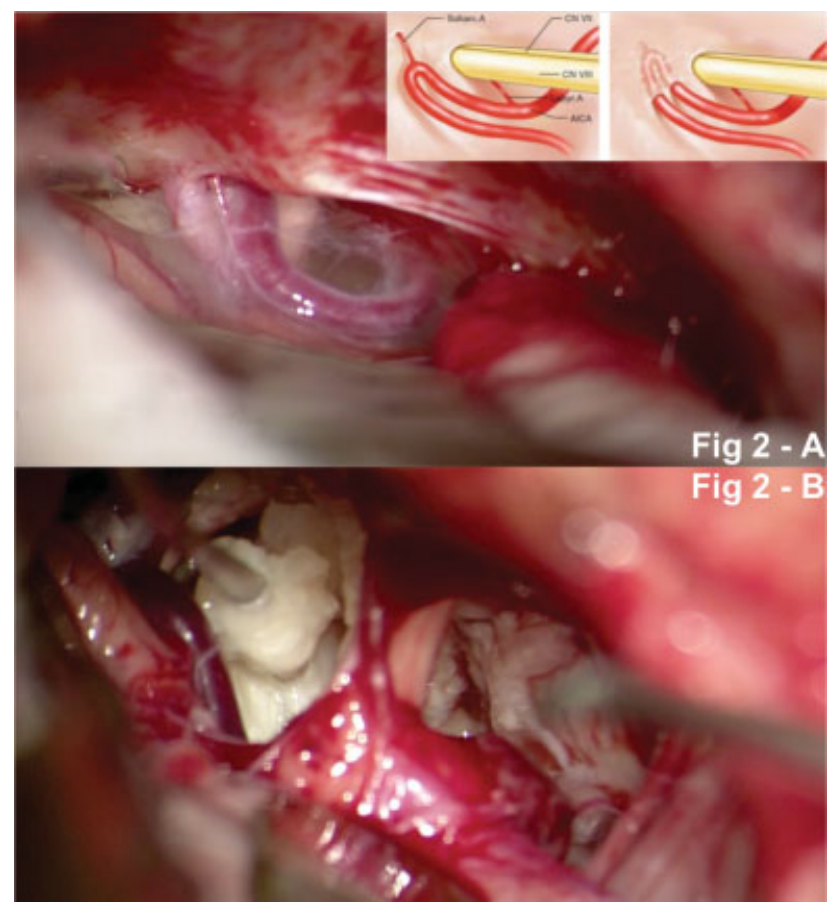

Fig. 2 Intraoperative imaging. (A) Intraoperative view showing the right anterior inferior cerebellar artery's (AICA's) loop embedded in the dura covering the subarcuate artery's exit. (B) View showing the epidermoid cyst tumor resection between the trigeminal nerve and the facial-vestibulocochlear bundle. 